Evidence of the need for such a utility model has, however, continued to accumulate with the increasing application of the electron microscope in many fields, and particularly sinc $\theta$ a number of uses have become sufficiently standardized as to become routine in nature, for example, in particle-size analysis. It was not surprising, therefore, that Metropolitan-Vickers should have followed its recent three-stage standard model ( $E M 3$ ) by designing a cabinet model ( $E M 4)$, which was shown last March at the exhibition in London of the Physical Society. More recently the Radio Corporation of America has announced the appearance of a new table model $(E M T)$. Both machines have a number of novel features, apart from the general simplification and reduction of cost which place them in a separate category from all other electron microscopes so far produced.

The EM4 is designed as a single unit, with hightension generator, smoothing circuits and vacuum pumps all contained in the same cabinet as the microscope proper and its controls. The $E M T$ consists of four separate units, allowing the microscope itself to be separately mounted along with the diffusion pump and some of the controls. The length of the column is kept to reasonable size (30 in.) by dispensing with a condenser lens and employing a moderate magnification (maximum, 6,000). The $E M 4$ achieves a column of about half this length, with a wide range of magnification (up to a maximum of 20,000), by using a projector lens having three magnetic gaps in place of the usual single gap. It thus acts as a four-stage microscope, instead of two-stage; the length of column is proportional to the sum of the separate magnifications, while the maximum magnification is given by their product. The resulting short column is fixed horizontally in the top of the cabinet, with the camera chamber at the front end. For visual observation, a magnetic deflecting field throws the image on to an inclined window above the camera. This innovation allows ready observation in a sitting position and simplicity of camera operation; the camera takes either roll film or plates. On the electrical side the EM4 incorporates dry rectifiers and voltage doubling in the high-tension supply, in order again to achieve compactness and reduction in cost, as compared with the conventional transformer-rectifying-valve system. In other respects the new model is a simplified version of the $E M 3$ instrument, apart from the inclusion of a beam-vibrator for ease of focusing.

The chief technical interest in the new American model, apart from the general design and mounting of the column, lies in the use of permanent magnets instead of energized windings for the objective and projector lenses. A machine of standard type was made some years ago using permanent magnets ${ }^{2}$, and von Borries recently described a simpler arrangement ${ }^{3}$; but no commercial model has previously departed from the use of coils for the magnetic lenses. In the Radio Corporation of America design the microscope column is near-vertical, with camera and viewingwindow at the bottom end. The magnetic circuit has two gaps, the upper forming the objective and the lower the projector lens. The magnification is fixed, there being no means of varying the reluctance of the circuit. Lens units giving magnifications of $1,500,3,000$ and 6,000 are available. As the positions of both lens and object are fixed, focusing is carried out by varying the accelerating voltage applied to the anode.
Both the new instruments are designed to operate at $50 \mathrm{kV}$. and to give a resolving power approaching $100 \mathrm{~A}$. The specimen and the photographic plates may be quickly changed, so that the rapid working desirable in routine applications is achieved. If the production models come up to the performance of the prototypes, they will represent an important step towards wider utilization of electron microscopical methods, especially at the technological level. In each case the price is indicated as being around $£ 2,000$, which is about half that of the standard models. The specified performance is certainly above half that shown by the latter; the $E M 4$, in particular, appears to have considerable flexibility as well as simplicity of operation.

V. E. COSSLETT

' Zworykin, V. K., end Hillier, J., J. App. Phys., 14, 658 (1943).

' Borries, B. von, Ruska, E., Krumm, J., and Müller, H. O., Naturviss., 28, 350 (1940)

s Borries, B. von, Koul. Z., 114, 164 (1949).

\section{BIOLOGY AND CONTROL OF COCCIDIOSIS}

THE range of parasitology nowadays is so wide that anyone choosing to work on it soon finds that he must not only have a very broad scientific training, but must also restrict his studies to one field, or even to one genus of parasitic animals. If he be interested in the Protozoa, for example, he may well find that the study of coccidiosis, or even the study of the economic aspects of this disease, will occupy all his life.

For anyone interested in this subject, the conference on coccidiosis convened by the New York Academy of Sciences* provides a sound synoptic view of it. Prefaced by a photograph of, and a short article by, Prof. E. E. Tyzzer, whose classic researches on the coccidia of hen chickens and other birds established the study of coccidiosis for the first time on a scientific basis, the proceedings of this conference cover most aspects of the biology of the species of coccidia considered, their host-parasite relationships and most of the methods used to control the diseases that they cause. The importance of their control is shown by the estimate worked out by one contributor that coccidiosis of poultry costs the United States ten million dollars a year, coccidiosis of cattle the same figure and coccidiosis of sheep half a million dollars, while the disease in goats, pigs, dogs, cats, rabbits and fur bearers adds another halfmillion dollars to the cost. The total cost of the disease in the United States is thus twenty-one million dollars a year.

It is impossible to summarize in a short space the wide range of this conference. It provides a valuable epitome of every aspect of the subject, and articles on the chemotherapy of the disease and on its nutritional aspects illustrate well the degree to which the parasitologist nowadays has to be, or has to try to be, a biochemist. Although most of the work is American, British research is represented by the article, contributed by Dr. C. Horton-Smith, on factors which influence the origin and course of epidemics of coccidiosis in poultry. The bibliographies appended to each article are a valuable feature of the publication.

- Annals of the New York Academy of Sciences, 52, Art. 4: Coccidiosis. By S. Blackett and forty-two other Authors. Pp. 429-624. (New 